\title{
Visual function and pattern visual evoked response in optic neuritis
}

\author{
E A C M SANDERS,' A C W VOLKERS, ' J C VAN DER POEL,' \\ AND G H M VAN LITH
}

From the 'Department of Neurology, University Hospital Leiden, ${ }^{2}$ Department of Biomedical Physics and Technology, Erasmus University, and the ${ }^{3}$ Eye Hospital, Rotterdam, The Netherlands

SUMmARY The disparity between clinical visual function and pattern visual evoked response (VER) was studied in 53 patients who had suffered an attack of optic neuritis (ON) more than six months before. The visual functions tested included Snellen visual acuity, colour vision, visual field, and contrast sensitivity. The effect of pattern presentation, check size, and luminance was tested by recording VERs with several stimulus configurations. VER amplitudes were found to be associated with the outcome of all four clinical tests, independently of check size, luminance, or the presentation method used. On the other hand VER latencies were hardly ever related to the results of any of the four clinical visual tests. These findings support the idea that VER amplitude provides information about visual spatial perception, while VER latency is more related to the extent of demyelination.

Optic neuritis (ON) usually gives a characteristic clinical picture with a fairly favourable short-term prognosis in the majority of patients. Normal vision, as measured with a Snellen chart test, is regained in $50-80 \%$ of the patients within six months. In some cases, however, ON produces long-lasting visual dysfunction. ${ }^{12}$

It has been suggested that Snellen chart tests are of questionable value for assessment of visual function in demyelinating disease, since they test only a restricted part of visual perception. ${ }^{3-5}$ The visibility of objects seems to be determined not only by how we see small details at high contrast, but also by how we discriminate between larger objects differing only slightly from their surroundings in colour and luminance. Hence some authors have suggested that the spatial contrast sensitivity function could be a more representative visual function in ON than the Snellen visual acuity. $\rightarrow-8$

There is little doubt that the visual evoked response (VER) to a black and white pattern is a valuable means of tracing optic nerve lesions in patients suspected of multiple sclerosis (MS) or optic neuritis. A delayed latency of the first major positive

Correspondence to A C W Volkers, Department of Biomedical Physics and Technology, PO Box 1738, 3000 DR Rotterdam, The Netherlands.
VER peak, found in $80-100 \%$ of clinically definite MS and ON patients, is a reliable indication of demyelination of the visual pathway. ${ }^{q 11}$

Neima and Regan ${ }^{12}$ tried to resolve some of the discrepancies between pattern VER and Snellen visual acuity by measuring the contrast sensitivity function. In general their results showed that abnormalities in the contrast sensitivity function could be used as a basis for predicting abnormalities in VER amplitude, while VER peak delay was less accurately predicted. The number of $\mathrm{ON}$ and MS patients they studied, however, was too small to permit meaningful statistical analysis. Neima and Regan ${ }^{12}$ used two different stimuli for VER determination (with check sizes of 45 and 11 minutes of arc). It is now generally accepted that the pattern VER response depends not only on check size but also on luminance and method of presentation..$^{1314}$

The object of the present study, like that of Neima and Regan, ${ }^{12}$ was to search for correspondences between VER recordings and four clinical visual parameters: visual acuity, contrast sensitivity function, colour vision, and visual field. A previous study revealed a statistically significant relation between these parameters. ${ }^{15}$ In the present investigation pattern visual evoked responses were recorded by different methods of stimulation in order to see 
how check size, luminance, and method of presentation changed the possible relation between VER and the four clinical parameters.

Visual function in 53 patients with optic neuritis (ON) was tested six months to 4.5 years after the acute attack. This investigation was particularly interesting in those cases where Snellen visual acuity exceeds $1 \cdot 0$, suggesting (nearly) complete recovery of visual function. It was hoped that the large battery of tests used would permit more accurate estimation of just how much visual function had improved. The size of the group studied (106 eyes) permitted meaningful determination of the statistical correlations between the various test results.

\section{Patients and methods}

\section{PATIENTS}

Fifty-three ON patients, treated between 1980 and 1983 at the Eye Hospital Rotterdam, were reexamined in 1984-5 for this study. The patient group included 40 females and 13 males. Their age at the time of this study varied from 17 to 54 years (mean 32 years). The diagnosis of $\mathrm{ON}$ was based on the criteria summarised by Glaser. ${ }^{\text {th }}$

Eleven patients had a relapse of $\mathrm{ON}$ within the period 1980-4. In eight of them the recurrence affected the contralateral, initially unaffected eye, while in the other three cases the relapse was on the ipsilateral side. Thus a total of 61 eyes had had a clinical ON attack at the time of this study. All 106 eyes investigated were classified on the basis of clinical status and Snellen visual acuity to: unaffected, affected with recovery, and affected without recovery on the basis of the following criteria:

\begin{tabular}{lll}
\hline Classification & $\begin{array}{l}\text { Clinical signs and } \\
\text { symptoms in the eye } \\
\text { during the ON attack }\end{array}$ & $\begin{array}{l}\text { Snellen visual acuity } \\
\text { during present study }\end{array}$ \\
\hline Unaffected $(\mathrm{n}=45)$ & No & $\geqslant 1 \cdot 0$ \\
Recovered $(\mathrm{n}=33)$ & Yes & $\geqslant 1 \cdot 0$ \\
Non-recovered $(\mathrm{n}=28)$ & Yes & $<1 \cdot 0$ \\
\hline
\end{tabular}

At the time of this investigation 29 of the 53 patients had clinical signs and symptoms of mild central nervous system involvement affecting structures other than the optic nerve, which was regarded as justifying the diagnosis of clinically probable or possible MS as defined by the criteria of Mc Alpine et al. ${ }^{17}$

\section{METHODS}

Visual evoked responses (VER) were obtained by checkerboard stimulation after correction of refractive errors.

Pattern reversal $(P R)$ was achieved with a projector system, the screen of which subtended a visual angle of $30^{\circ}$. Fast pattern reversal was obtained with the aid of an electromagnetically activated mirror. ${ }^{18}$ The squares of side $0.5^{\circ}$ forming the pattern were presented with a mean luminance of $10 \mathrm{~cd} / \mathrm{m}^{2}$, those of side $1^{\circ}$ with luminances of $10 \mathrm{~cd} / \mathrm{m}^{2}$ and $100 \mathrm{~cd} / \mathrm{m}^{2}$. Contrast between the checks was approximately $80 \%$ and the reversal frequency $1 \mathrm{~Hz}$ (i.e., 2 reversals per second).

Pattern (intermittent) presentation $(P P)$ was accomplished with a TV monitor screen; the visual angle subtended was $16^{\circ}$. In this method the pattern was presented for $39 \mathrm{~ms}$ in each viewing cycle, with a repetition frequency of $2 \mathrm{~Hz}$. The mean luminance of the screen both during and outside the pattern presentation was kept constant at $45 \mathrm{~cd} / \mathrm{m}^{2}$. The various patterns used had a check size (minutes of arc) and contrast respectively of $80^{\prime} / 80 \%, 400^{\prime} / 40 \%$, $20^{\prime} / 20 \%, 10^{\prime} / 10 \%$, and $10^{\prime} 5 \%$.

In both methods the patients were asked to look at a fixation spot at the centre of the stimulus field. In accordance with Halliday's method" VERs were recorded with two occipital electrodes placed $2.5 \mathrm{~cm}$ and $5 \mathrm{~cm}$ above the inion (external occipital protuberance). A midfrontal electrode $12 \mathrm{~cm}$ above the nasion served as the reference electrode; the ground electrode was attached to the ear lobe. Two derivations were made, from each of the electrodes above the inion against the midfrontal one.

The bandwidth of the amplifiers was 0.166 to $70 \mathrm{~Hz}$. In general 128 responses were averaged in all VER determinations. The output was fed simultaneously to an oscilloscope with a sweep time of $500 \mathrm{~ms}$ and to a minicomputer with a sampling time of $3.9 \mathrm{~ms}$. With these techniques responses of $3 \mu \mathrm{V}$ or more could be discerned from the noise level. The VER thus obtained was recorded on an X-Y plotter.

Normal values were obtained from 47 healthy control subjects. For the latency the 90th percentile was taken as the upper limit of normal; for amplitude the 90th percentile was taken as the lower limit of normal.

The contrast sensitivity function was determined with equipment described previously. ${ }^{1519}$ The automatic, microprocessor-controlled measuring procedure is based on a modified von Bekesy tracking method, ," which may be briefly described as follows. The stimulus (a vertical sinusoidal grating) is presented on a television monitor. The patient is asked to decrease or increase the contrast of the grating by pushing or releasing a button so as to hold it at his or her perception threshold. After eight reversals the average contrast is calculated and plotted, after which the spatial frequency is increased by a factor 2 . The contrast sensitivity is determined over the range 
from $0 \cdot 1$ to $25 \cdot 6$ cycles per degree (c/deg). The height of the stimulus is $5^{\circ}$ and its width is $32^{\circ}$ for the spatial frequency range from 0.1 to $0.4 \mathrm{c} / \mathrm{deg}$ (at a viewing distance of $50 \mathrm{~cm}$ ) and $8^{\circ}$ for spatial frequencies from 0.8 to $25.6 \mathrm{c} / \mathrm{deg}$ ( $200 \mathrm{~cm}$ viewing distance). To prevent after-images the grating is counterphased every $0.6 \mathrm{~s}$. The mean luminance of the stimulus is $5 \mathrm{~cd} / \mathrm{m}^{2}$.

The stimulus is defined as: $\mathrm{C}=\left(\mathrm{L}_{\max }-\mathrm{L}_{\min }\right) /$ $\left(\mathrm{L}_{\max }+\mathrm{L}_{\min }\right)$, where $\mathrm{L}_{\max }$ and $\mathrm{L}_{\min }$ are the luminances of the light and dark 'bars' respectively. The contrast sensitivity is defined as the reciprocal of the contrast at threshold: $\mathrm{CS}=1 / \mathrm{C}(\mathrm{thr})$.

The normal contrast sensitivity function was determined in 26 normal subjects ( 52 eyes) with the same age range as the $53 \mathrm{ON}$ patients. The number of contrast determinations per eye was reduced to only three by averaging contrast sensitivities in the low $(0 \cdot 1-0 \cdot 4 \mathrm{c} / \mathrm{deg})$, medium $(0 \cdot 8-3 \cdot 2 \mathrm{c} / \mathrm{deg})$, and high $(6 \cdot 4-25 \cdot 6 \mathrm{c} / \mathrm{deg})$ spatial frequency ranges. A decrease of more than 2 standard deviations below the normal (average) contrast sensitivity for low, medium, or high spatial frequencies is considered abnormal.

Visual acuity was determined with a Snellen chart and expressed as the reciprocal of the mean angle of resolution in minutes of arc. A visual acuity of 1.0 or more was considered normal.

Colour vision was examined under standardised conditions, with the Hardy Rand Rittler (HRR) and desaturated panel D-15 tests. Normal values were obtained from 25 control subjects, classified according to the classification of François and Verriest. ${ }^{21}$

Visual fields were determined with the computerised Octopus display. Visual field disorders were classified as central or paracentral absolute or relative defects. ${ }^{22}$

Routine clinical ophthalmological and neurological examination was performed on all 53 patients.

Statistical analysis was performed with the SPSS computer program. Differences between sets of experimental data were tested for significance by the Wilcoxon, $\chi^{2}$, Kendall's $b$ and t-c tests, and regression analysis.

Table 1 Distribution of visual function abnormalities

\begin{tabular}{|c|c|c|c|c|c|c|}
\hline \multicolumn{2}{|c|}{ VER stimulus configuration } & Low amplitude $(\mu V)$ & High latency (ms) & $\begin{array}{l}\text { Unaffected eyes } \\
(n=45)\end{array}$ & $\begin{array}{l}\text { Recovered eyes } \\
(n=33)\end{array}$ & $\begin{array}{l}\text { Non-recovered } \\
\text { eyes }(n=28)\end{array}$ \\
\hline \multicolumn{7}{|c|}{ With PPpresentation: } \\
\hline $\begin{array}{l}\text { Check size } \\
\text { (min of arc) }\end{array}$ & $\begin{array}{l}\text { Contras } \\
(\%)\end{array}$ & & & & & \\
\hline \multirow[t]{2}{*}{80} & 80 & $<8 \cdot 6$ & & 1 & 2 & 17 \\
\hline & & & $>192$ & 9 & 12 & 14 \\
\hline \multirow[t]{2}{*}{40} & 40 & $<12 \cdot 5$ & & 5 & 8 & 21 \\
\hline & & & $>195$ & 12 & 17 & 19 \\
\hline \multirow[t]{2}{*}{20} & 20 & $<8 \cdot 2$ & & 11 & 16 & 22 \\
\hline & & & $>222$ & 12 & 11 & 20 \\
\hline \multirow[t]{2}{*}{10} & 10 & $<5 \cdot 5$ & & 12 & 14 & 23 \\
\hline & & . & $>198$ & 16 & 18 & 25 \\
\hline \multicolumn{7}{|c|}{ With PR presentation: } \\
\hline \multirow{2}{*}{$\begin{array}{l}\text { Check size } \\
\text { (min of arc) } \\
60\end{array}$} & 130 & $<7.9$ & & 11 & 16 & 22 \\
\hline & & & $>111$ & 21 & 23 & 23 \\
\hline \multirow[t]{2}{*}{60} & 13 & $<6.9$ & & 8 & 14 & 18 \\
\hline & & & $>124$ & 11 & 19 & 18 \\
\hline \multirow[t]{2}{*}{30} & 13 & $<6 \cdot 3$ & & 14 & 16 & 22 \\
\hline & & & $>122$ & 14 & 22 & 25 \\
\hline \multicolumn{7}{|c|}{ Total abnormalities in: } \\
\hline \multicolumn{4}{|l|}{ VER } & 37 & 32 & 22 \\
\hline \multicolumn{2}{|l|}{$\mathrm{CS}$} & & & 12 & 13 & 26 \\
\hline \multicolumn{2}{|c|}{ Visual field } & & & 6 & 6 & 20 \\
\hline \multicolumn{2}{|c|}{ One or more visual } & & No. & 41 & 33 & 28 \\
\hline \multicolumn{2}{|c|}{ functions tested: } & & $\%$ & 91 & 100 & 100 \\
\hline
\end{tabular}

Row 1 tells us that, in the VER test using PP stimuli with, for example, check-size 80 ' of arc and contrast $80 \%$, amplitudes less than $8.6 \mu \mathrm{V}$ were taken as abnormally low. Such low amplitudes were found in one out of 45 'unaffected', two out of 33 'recovered', and 17 out of 28 'nonrecovered' eyes. Row 2 tells us that in the same test latencies greater than $192 \mathrm{~ms}$ were high and found in 9,12 , and 14 eyes in the respective groups. 


\section{Results}

VISUAL FUNCTION AFTER OPTIC NEURITIS

The results of clinical visual testing in this patient group have been described in greater detail elsewhere. ${ }^{15}$ A summary of these results, in combination with those obtained by pattern reversal (PR) and pattern (intermittent) presentation (PP) VER, is presented in Table 1.

A high percentage $(96 \%)$ of non-recovered eyes showed at least one abnormal VER (either PP or $\mathrm{PR}$ ). In only one eye did the VER results fall within the normal range. A similar high percentage of VER abnormalities (97\%) was obtained in all affected eyes that had recovered $(n=33)$. The most surprising result was that no fewer than 37 of the 45 unaffected eyes $(82 \%)$ showed VER abnormalities (in latency, amplitude, or a combination of the two). A very small proportion of abnormal results may be ascribed to the $2 \%$ of normal subjects we may expect to find outside the normal range; this does not markedly affect the fact that the percentage of abnormalities found by PP (32\%) and PR (56\%) is strikingly high.

As was to be expected (and has been reported previously, ${ }^{15}$ all other visual tests gave the highest percentage of abnormal results in the non-recovered eyes and lower percentages in recovered and unaffected eyes.

The absolute number of VER abnormalities tends to be higher in those patients who had developed signs and symptoms outside the visual pathways, but the difference was not statistically significant (not shown in the tables). This tendency is in accordance with the previous findings for the four clinical visual tests. $^{15}$

\section{VISUAL EVOKED RESPONSE AND CONTRAST} SENSITIVITY FUNCTION

In 47 eyes VER abnormalities (found by PP and/or PR) were observed in combination with an abnormal contrast sensitivity function. In six eyes both VER and contrast sensitivity were normal. This indicates that the results of VER and contrast sensitivity testing in all eyes investigated $(n=106)$ overlap by no more than $50 \%$ (Table 2 ). The lack of a statistically significant association between overall VER and contrast sensitivity testing is disappointing (Table 2). We therefore examined these results in greater detail to see whether this could reveal some meaningful relationships between the two types of tests.

Consideration of VER amplitude and latency separately yields a more differentiated picture than that obtained by regarding overall results only. In Fig. 1 correlation coefficients are plotted for each VER stimulus and the contrast sensitivity function in the three frequency ranges considered. We use
Table 2 Relation between incidences of normal and abnormal VER and CS

\begin{tabular}{|c|c|c|c|c|}
\hline \multicolumn{2}{|c|}{ Abnormal VER } & \multicolumn{3}{|c|}{ Contrast $\sim$ sensitivity $\sim$ function } \\
\hline $\begin{array}{l}\text { With PP } \\
\text { stimulus }\end{array}$ & $\begin{array}{l}\text { With PR } \\
\text { stimulus }\end{array}$ & Normal & Abnormal & Total \\
\hline+ & - & 8 & 1 & 9 \\
\hline- & + & 14 & 4 & 18 \\
\hline+ & + & 27 & 42 & 69 \\
\hline- & - & 6 & 4 & 10 \\
\hline Total & & 55 & 51 & 106 \\
\hline
\end{tabular}

$A+$ in the first two columns refers to abnormal VER with the stimulus type indicated; $a-$ in these columns refers to normal values. Thus row 3 tells us that 69 of the total 106 eyes showed VER abnormalities with both PP and PR stimuli. Of these 69 eyes 42 also showed CS abnormalities; the other 27 had normal CS (see text).

$\mathrm{CS}(\mathrm{L})$ to denote the contrast sensitivity function in the low frequency range, $\mathrm{CS}(\mathrm{M})$ for that in the medium frequency range, and $\mathrm{CS}(\mathrm{H})$ for that in the high frequency range. The results can be summarised as follows: there is a significant association (with alpha one-sided $=0.05 ; n=53$ ) between $\mathrm{CS}(\mathrm{L})$, $\mathrm{CS}(\mathrm{M})$, and $\mathrm{CS}(\mathrm{H})$ on the one hand and VER amplitude on the other, while VER latency is not significantly related to CS in any of these frequency ranges with the exception of the relation found between $10^{\prime}-10 \%$ latency on the one hand and CS(L) and $\mathrm{CS}(\mathrm{M})$ on the other. The association between $\mathrm{CS}(\mathrm{L})$ and $\mathrm{CS}(\mathrm{M})$ and VER amplitude is stronger for middle sized checks (PP: $200^{\prime}-20 \%$ and $400^{\prime}-40 \%$ ) than for the smaller $\left(10^{\prime}-10 \%\right)$ and larger $\left(80^{\prime}-80 \%\right)$ checks, while $\mathrm{CS}(\mathrm{H})$ and reduced VER amplitude is strongest for small check sizes $\left(10^{\prime}-10 \%\right)$.

\section{VISUAL ACUITY, COLOUR VISION, AND VISUAL} FIELD

An association between reduced visual acuity and abnormalities in visual field and colour vision on the one hand and contrast sensitivity abnormalities on the other has been previously reported. ${ }^{\text {is }}$ In the present study we were interested in the relation between VER results and those of the above mentioned clinical tests. Since colour vision and visual field are assessed as 'normal' or 'abnormal', we cannot express the association between these clinical test results and the VER results in terms of correlation coefficients. The quantity plotted in Fig. 2, middle and right columns, is therefore the 'phi' coefficient, which does provide a measure of such associations.

Inspection of Fig. 2 shows that reduced VER amplitude (in both PR and PP tests) is associated with abnormal results in all four clinical tests, whereas VER latency does not show any such association. 


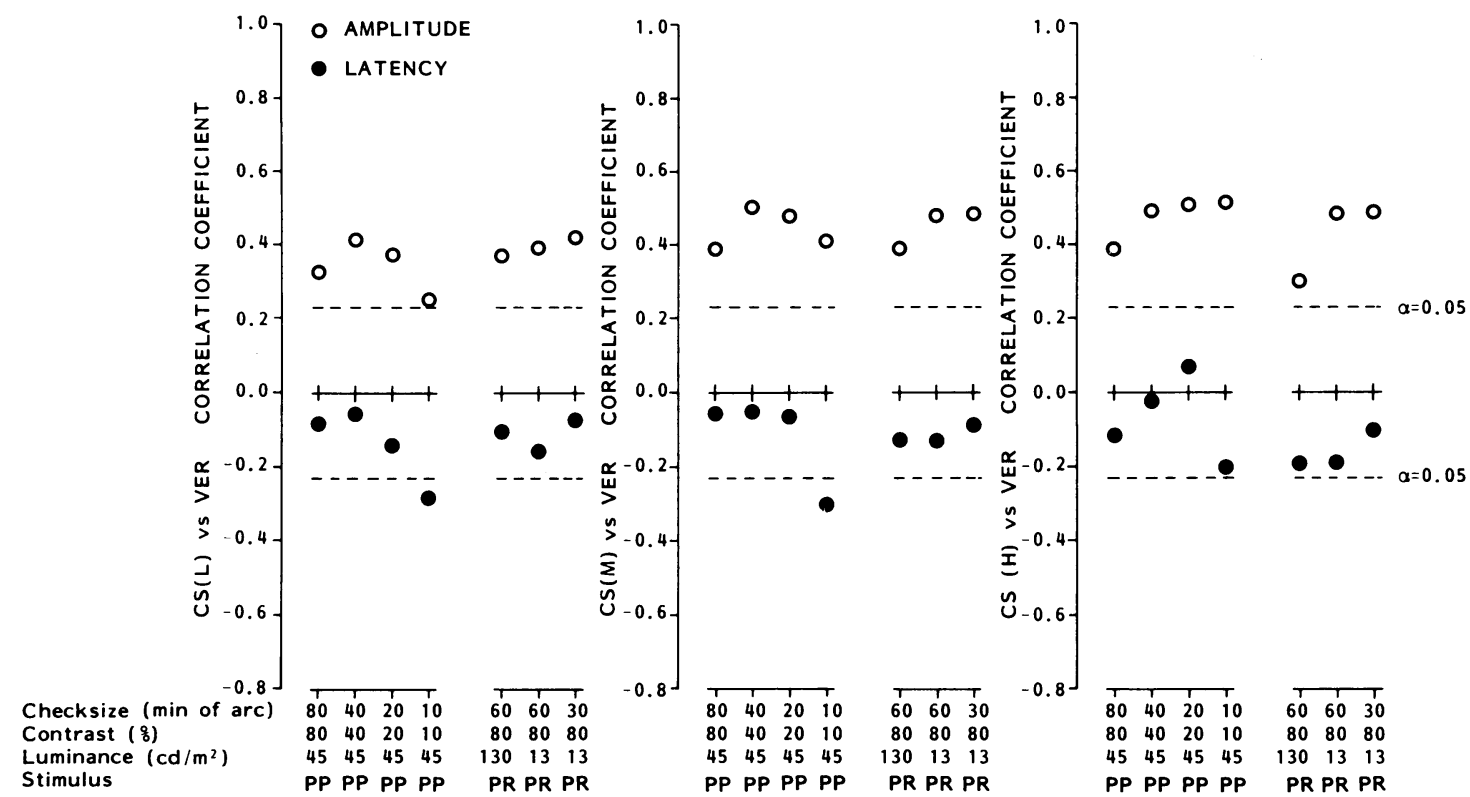

Fig. 1 Association between contrast sensitivity for several frequency ranges and visual evoked response (VER) for various stimulus configurations (details given under each graph). The frequency ranges are: Left: low $(C S(L) ; 0 \cdot 1-0 \cdot 4$ cldeg). Middle: medium (CS $(M) ; 0 \cdot 8-3 \cdot 2 \mathrm{c} / \mathrm{deg})$. Right: high $(C S(H) ; 6 \cdot 4-25 \cdot 6 \mathrm{cldeg})$. The correlation coefficients between the broken lines are not significantly different from zero ( $\chi^{2}$ double-sided $\left.=0 \cdot 10 ; n=53\right)$.
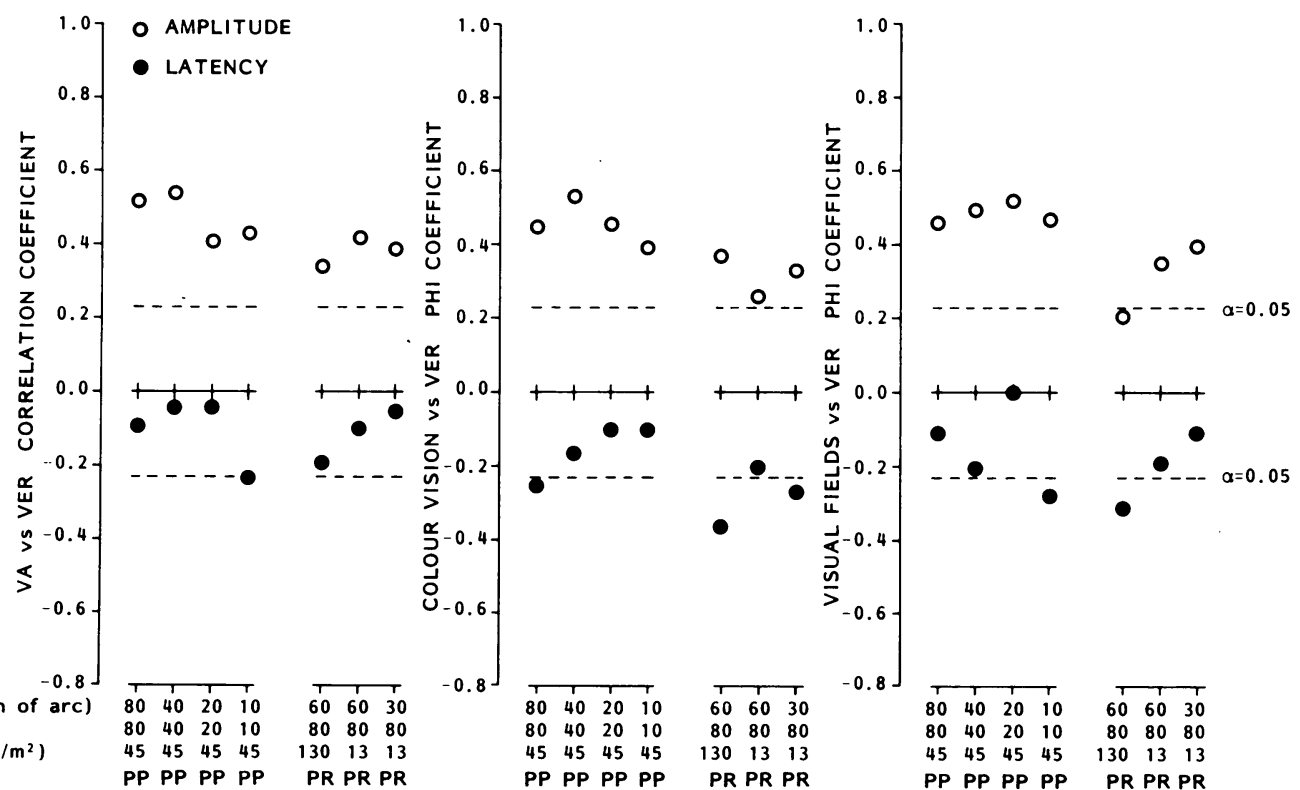

Fig. 2 Association between several clinical visual function tests and visual evoked response (VER) for various stimulus configurations (details given under each graph). The visual function tests are: Left: Snellen visual acuity (VA). Middle: colour vision (PDI5 or HRR test). Right: visual fields (determined with the computerized Octopus display). The correlation coefficients (left) or the phi coefficients (middle and right) between the broken lines are not significantly different from zero. 


\section{Discussion}

\section{COMPARISON WITH PREVIOUS STUDIES}

It is well known that, in optic neuritis (ON), visual evoked response (VER) is not closely related to sensory visual loss as determined with Snellen charts. ${ }^{224}$ It has been suggested that the 'hidden visual loss' which presumably exists in such cases may be detected by psychophysical tests which provide a measure of contrast sensitivity, visual field, and colour vision..$^{15256}$ Comparison of the results of these tests with VER measurements often revealed a considerable degree of overlap in ON or MS patients. ${ }^{27-31}$ However, differences become apparent if VER latency and amplitude are considered separately ${ }^{32}$ Neima and Regan ${ }^{12}$ made this distinction in their attempt to correlate visual acuity, contrast sensitivity, and VER findings in ON and MS patients. They reported that reduced contrast sensitivity at high spatial frequencies was associated with reduced visual acuity and amplitude reductions in small-check VER tests. In all eyes where the contrast sensitivity was reduced at all spatial frequencies they found amplitude reductions in both small-check and large-check VERs, and diminished Snellen visual acuity. In the present study Snellen visual acuity and the other psychophysical test results were compared with VER latency and amplitude separately in all cases.

\section{VISUAL EVOKED RESPONSE AND VISUAL TESTS} IN ON

In general Figs. 1 and 2 indicate that VER amplitude is correlated with the results of all three psychophysical tests and with the Snellen visual acuity, while VER latency shows no such correlation.

In this study VER recordings were based on stimulation by checkerboard patterns with a variety of check sizes and luminance contrasts. Moreover, as we have seen, two different methods of checkerboard presentation were used (PR and PP). As mentioned in the introduction to the present paper, this wide variety of stimulus parameters was adopted in the hope that it would allow more precise detection of possible associations between clinical visual tests and VER. We found that VER results in response to pattern presentation of medium-sized checks with $40 \%$ luminance contrast gave the highest correlation $(0 \cdot 4-0 \cdot 55)$ with contrast sensitivity for all spatial frequency ranges as well as with colour vision and with visual acuity.

Of particular interest was the finding that all but one of the $33 \mathrm{ON}$ eyes classified as 'recovered' at the time of this study (visual acuity $>1 \cdot 0$ ) showed a VER abnormality (either in amplitude, latency, or combination of the two). Seventeen of these 32 eyes also gave abnormal results in one or more psychophysical tests. This indicates that VER recording is the most sensitive test for detecting hidden visual disturbance in ON (or MS). This conclusion is supported by the results of all tests of the 45 unaffected eyes. In our previous report ${ }^{15}$ we stated that $67 \%$ of these eyes gave abnormal results in one or more psychophysical tests. When we add the results of our VER investigations, we find over $91 \%$ of all unaffected eyes show abnormality in at least one test.

Our finding in the present study that reduced Snellen visual acuity and defects of colour vision and visual field are associated with diminished VER amplitude, but not with prolonged VER latency, is in line with our previous report that contrast sensitivity is associated with visual acuity, visual field, and colour vision. ${ }^{15}$ It is therefore not surprising that contrast sensitivity is related to VER amplitude and not to VER latency.

One of Neima and Regan's suggested explanations ${ }^{12}$ for the discrepancy between Snellen visual acuity and VER in ON concerns the difference between the eye's ability to see fine detail (measured by what we call $\mathrm{CS}(\mathrm{H})$ ) and coarse detail (measured by VER with large check stimulation). This would indicate that the association found between VER and contrast sensitivity would depend mainly on check luminance and/or check size. However, in the present study the associations between the results of clinical visual tests and VER recordings hardly depended on stimulus configuration at all.

A more fundamental difference between the physiological mechanisms underlying visual acuity and VER latency must thus be at the root of the above-mentioned discrepancies. The VER latency is assumed to be related to the conduction velocity within the visual neural system. Chronic demyelination of the optic nerve will reduce the conduction velocity and hence prolong VER latency. However, there are indications that increases in VER latency can also be due to neurotransmitter deficiency. ${ }^{33}{ }^{34}$ In contrast with this, VER amplitude is associated with all the clinical parameters (including Snellen visual acuity) used in this study and must therefore be related to the actual functional state of the retina and optic nerve fibres as regards spatial perception. This supports our assumption that VER latency and VER amplitude reflect different aspects of the visual system. The reason for the discrepancies between Snellen visual acuity (and other clinical visual parameters) and VER is thus perhaps to be sought in factors related to VER latency.

The authors are indebted to Dr R H Bathgate, Eindhoven, for his final comments, and Mrs Ch J Th Sanders-Bozon for accurate prepartion of the manuscript. 


\section{References}

1 Bradley WG, Whitty CW. Acute optic neuritis: its clinical features and their relation to the prognosis for recovery of vision. J Neurol Neurosurg Psychiatry 1967: 30: 531-7.

2 McAlpine D. Optic retrobulbar ncuritis. In: McAlpine D, Lumsden CE, Acheson ED, eds. Multiple sclerosis: a reappraisal. Edinburgh: Churchill Livingstone, 1970: 148-63.

3 Campbell FW, Maffei L. Contrast and spatial frequency. Scientific American 1974; 231: 106-14.

4 Braddick O. Spatial frequency analysis in vision. Nature 1981; 291: 9-11.

5 Bodis Wollner J, Hendley CD, Mylin LH, Thornton J. Visual evoked potentials and visuograms in multiple sclerosis. Ann Neurol 1979; 5: 40-7.

6 Regan D, Silver R, Murray TJ. Visual acuity and contrast sensitivity in multiple sclerosis: hidden visual loss. Brain 1977; 100: $563-79$.

7 Zimmern RL, Campbell FW, Wilkinson JMS. Subtle disturbances of vision after optic neuritis elicited by studying contrast sensitivity. J Neurol Neurosurg Psychiatry 1979; 42: 407-12.

8 Sanders EACM, Volkers ACW, van der Poel JC, van Lith GHM. Spatial contrast sensitivity function in optic neuritis. Neuroophthalmology 1984; 4: 255-9.

9 Halliday AM, McDonald WI, Mushin J. Visual evoked responses in optic neuritis. Lancet 1972; i: 982.

10 Richey ET, Kooi KA, Tourtelotte WW. Visually evoked responses in multiple sclerosis. J Neurol Neurosurg Psychiatry 1971: 34: 275-80.

11 Asselman P, Chadwick DW, Marsden CD. Visual evoked responses in the diagnosis and management of patients suspected of multiple sclerosis. Brain 1975; 98: 261-82.

12 Neima D, Regan D. Pattern visual evoked potentials and spatial vision in retrobulbar neuritis and multiple sclerosis. Arch Neurol 1984; 41: 198-201.

13 Nilsson BY. Visual evoked responses in multiple sclerosis: comparison of two methods for pattern reversal. J Neurol Neurosurg Psychiatry 1978; 41: 499-504.

14 Riemslag FCC, Spekreijse H, van Walbeek H. Pattern evoked potential diagnosis of multiple sclerosis: a comparison of contrast stimuli. Adv Neurol 1982; 32: 417-26.

15 Sanders EACM, Volkers ACW, van der Poel JC, van Lith GHM. Estimation of visual function after optic neuritis: a comparison of clinical tests. BrJ Ophthalmol 1986; 70: 918-24.

16 Glaser JS. Topical diagnosis: prechiasmal visual pathways. In: Duane TD, Jaeger EA, eds. Clinical ophthalmology. Philadelphia: Harper and Row, 1982; 2: 1-72.

17 McAlpine D, Lumsden CE, Acheson ED, eds. Multiple sclerosis: a reappraisal. Edinburgh: Churchill Livingstone, 1972.

18 Cobb WA, Morton HB, Ettlinger G. Cerebral potentials evoked by pattern reversal and their suppression in visual rivalry. Nature 1967; 216: 1123-5.

19 Keemink CJ, van der Wildt GJ, van Deursen JBP. Microprocessor controlled contrast sensitivity measurements. Med Biol Eng Comput 1979; 17: 371-8.

20 Von Bekesy G. A new audiometer. Acta Otolaryngol (Stockh) 1947; 35: 411-22.

21 François J, Verriest G. Les dyschromatopsies. Ann Oculist (Paris) 1957; 190: 713, 812,893.

22 Walsh TJ, Visual field defects. In: Walsh TJ, ed. Neuroophthalmology. Clinical signs and symptoms. Philadelphia: Lea and Febiger, 1985: 434-69.

23 Regan D, Bartol S, Murray TJ, Beverley KI. Spatial frequency discrimination in normal vision and in paticnts with multiple sclerosis. Brain 1982; 105: 735-54.

24 Regan D, Raymond J, Ginsburg AP, Murray TJ. Contrast sensitivity, visual acuity and discrimination of Snellen letters in multiple sclerosis. Brain 1981; 104: 37: 333-50.

25 Patterson H, Heron JR. Visual field abnormalities in multiple sclerosis. J Neurol Neurosurg Psychiatry 1980; 43: 205-8.

26 Pinckers A, Hardus P, Nabbe B. Comparison of visual evoked cortical potentials and color vision in presumed demyelinating disease. Neuroophthalmology 1983; 3: 161-5.

27 Bynke H, Rosen I, Sandberg-Wollheim M. Correlations of visual evoked potentials, ophthalmological and neurological findings after unilateral optic neuritis. Acta Ophthalmol (Kbh) 1980; 58: 670-87.

28 Kirkham TH, Coupland SG. Multiple regression analysis of diagnostic predictors in optic nerve disease. Can J Neurol Sci 1981; 8: 67-72.

29 Bucrki E. Visuell evozicrte Potentiale, Kontrastempfindlichkeit und Farbsinn bei Patienten mit Neuritis Nervi Optici und bei Multipler Sclerose. Klin Monatsbl Augenheilkd 1981; 179: 161-8.

30 Ellenberger $\mathrm{C}$, Ziegler SD. Visual evoked potentials and quantitative perimetry in multiple sclerosis. Ann Neurol 1977; 1: $561-4$.

31 Heron JR, Regan D, Milner BA. Delay in visual perception in unilateral optic atrophy after retrobulbar neuritis. Brain 1974; 97: 69-78.

32 Regan D, Milner BA, Heron JR. Delayed visual perception and delayed evoked potentials in the spinal form of multiple sclerosis and retrobulbar neuritis. Brain 1976; 99: 43-63.

33 Wray SH. Optic neuritis. In: Lessel S, van Dalen JTW, eds. Neuroophthalmology. Part II. Amsterdam: Excerpta Medica, 1982: 13-31.

34 Gawel MJ, Das P, Vincent S, Clifford Rose F. Visual and auditory evoked responses in patients with Parkinson's disease. J Neurol Neurosurg Psychiatry 1981; 44: 227-32.

Accepted for publication 9 September 1986. 